\title{
AXOTOMY-INDUCED PARALLEL INCREASES IN ELECTRICAL AND DYE COUPLING BETWEEN IDENTIFIED NEURONS OF HELISOMA ${ }^{1}$
}

\author{
A. DON MURPHY, R. D. HADLEY, ${ }^{2}$ AND S. B. KATER ${ }^{3}$ \\ Department of Zoology, University of Iowa, Iowa City, Iowa 52242
}

Received November 2, 1982; Revised February 9, 1983; Accepted February 9, 1983

\begin{abstract}
The electrically coupled buccal ganglion neurons $4 \mathrm{R}$ and $4 \mathrm{~L}$ of the snail, Helisoma, display predictable plasticity. The strength of the electrotonic synapse between them increases significantly following axotomy. Synaptic strength was assayed by measurements of electrical coupling coefficients and by assessment of dye coupling (passage of dye into the uninjected neuron) following injection of Lucifer Yellow $\mathrm{CH}$ into one neuron. Within 3 to 5 days, axotomy induced an increase in electrical coupling coefficients between neurons $4 \mathrm{R}$ and $4 \mathrm{~L}$ from $0.54 \pm 0.11(n=13)$ in normal preparations to $0.72 \pm 0.14(n=24)$. A parallel axotomy-induced increase in the probability of dye coupling occurred. Only $27 \%(n=27)$ of normal neuron 4 pairs were dye coupled, compared with $87 \%$ ( $n=$ 15) of axotomized neuronal pairs.

Irrespective of treatment, electrical and dye measurements in the same neuron 4 pairs showed a consistent correlation between the magnitude of the electrical coupling coefficients and the probability of detectable dye coupling. No dye coupling was observed in neuronal pairs with electrical coupling coefficients less than 0.50 . Dye coupling always occurred when coupling coefficients were greater than 0.70 . Sixty-seven percent of neuronal pairs with intermediate coupling (0.50 to 0.70$)$ coefficients displayed dye coupling. The results show that axotomy evokes a predictable enhancement of communication at an "identified" electrotonic synapse and suggests that electrical and dye coupling are mediated by similar mechanisms.
\end{abstract}

Highly stereotyped synaptic interactions occur between identified neurons in different individuals of a given species (e.g., Kandel, 1976). A priori this reproducibility of neural circuitry could reflect a rigid and fixed set of neuronal properties resulting from highly constrained developmental programming. More likely, however, the connectivity of mature neuronal circuits may represent a steady-state condition in which neurons also respond to extrinsic conditions. There is evidence, for example, of hormonally regulated seasonal remodelling of circuitry underlying song production in birds (DeVoogd and Nottebohm, 1981; Nottebohm, 1981), and remodelling of synaptic interactions of identified neurons occurs during metamorphosis of insects (Levine and Truman, 1982). Plasticity of neuronal circuitry, however,

\footnotetext{
${ }^{1}$ This work was supported by United States Public Health Service Grants NS 15350, NS 18819, and AM 19858. We wish to thank Drs. C. Cohan and $R$. Wong for their valuable comments on the manuscript. We also thank Paul Gade for his expert technical and photographic assistance.

${ }^{2}$ Current address: Department of Physiology and Biophysics, Washington University Medical School, $660 \mathrm{~S}$. Euclid Avenue, St. Louis, MO 63110.

${ }^{3}$ To whom correspondence should be addressed.
}

surely involves not only the formation and elimination of synapses but also changes in efficacy of synaptic strength. In general, little is known about the degree of functional rigidity of individual synapses. To ascertain the limits of normal or experimentally manipulated communication between specific neurons, quantitative assessments of synaptic strength are required. Electrotonic synapses between identified neurons may provide a good opportunity for initial assessments of synaptic plasticity. For a particular identified electrotonic synapse, one may obtain quantitative electrophysiological measurements of electrical coupling. In addition, dye coupling can provide a complementary assay of communication.

Identified neurons in adult snails of Helisoma display a number of predictable changes in communication within $48 \mathrm{hr}$ of axotomy. Among the most striking of these changes is the de novo formation of entirely new electrical synapses, some of which are transient and some of which have long-term stability (Bulloch and Kater, 1981, 1982; Hadley and Kater, 1983). An interesting suggestion from previous studies is that some of the observed changes in connectivity (e.g., between cyberchron interneurons and protractor motoneurons) may represent the strengthening of very weak extant connections (cf. Granzow, 1979, and Bulloch et al., 1980). We therefore exam- 
ined quantitatively the effects of axotomy on the flexibility of normal communication between a pair of identified neurons (neurons $4 \mathrm{R}$ and $4 \mathrm{~L}$ ) with relatively strong electrotonic coupling. The present paper demonstrates that axotomy results in a marked increase both in the strength of electrical coupling and in the probability of Lucifer Yellow dye coupling between neurons 4R and 4L. Furthermore, a definitive relationship between electrical and dye coupling is established such that dye coupling is correlated not with the presence or absence of electrical coupling but with its magnitude.

\section{Materials and Methods}

Experimental animals and culture conditions. Inbred albino laboratory stocks of the pond snail, Helisoma trivolvis, were used. Specimens used for measurements of "normal" electrical and dye coupling were acutely dissected (Kater and Kaneko, 1972). The buccal ganglia were pinned to Sylgard pads, for electrophysiological measurements and dye injections. For studies of axotomy-induced effects we utilized the Helisoma blood hanging drop method for in vitro culture (Hadley and Kater, 1983). Blood collection and dissections for in vitro culture were under aseptic conditions and cultures were maintained at room temperature with buccal ganglia suspended in blood drops in sterile humidified chambers for 3 to 5 days. Neurons $4 \mathrm{R}$ and $4 \mathrm{~L}$ were axotomized by crushing the esophageal nerve trunks with no. 5 Dumont forceps. Unless otherwise noted, crushes were made near the buccal ganglia because such crushes evoke extensive central neuritic sprouting, whereas distal axotomy evokes primarily peripheral sprouting (Murphy and Kater, 1980a; Bulloch and Kater, 1982; Hadley et al., 1982).

Electrophysiology and dye injections. Standard electrophysiological techniques were used. Glass, fiber-filled microelectrodes were filled with $4 \mathrm{M}$ potassium acetate (DC resistance $=20$ to 40 megohms) and were connected by a unity gain, high input impedance amplifier to an oscilloscope and, for permanent records, a Brush 220 chart recorder. A virtual ground current monitor was employed. Neuron penetration was under visual control through a stereomicroscope while the preparation was bathed in standard Helisoma physiological saline (in mM: $\mathrm{NaCl}, 51.3 ; \mathrm{KCl}, 1.7 ; \mathrm{CaCl}_{2}, 4.1 ; \mathrm{MgCl}_{2}, 1.5 ;$ HEPES buffer, 5 at $\mathrm{pH} 7.3)$.

For electrical coupling measurements a balanced Wheatstone bridge circuit (Merickel et al., 1977) was used for simultaneous current injection and voltage monitoring with a single microelectrode. Coupling measurements were made after exchanging the standard physiological saline with low $\mathrm{Ca}^{2+} / \mathrm{high} \mathrm{Mg}^{2+}$ saline $\left(0 \mathrm{mM} \mathrm{Ca}{ }^{2+}\right.$, $15 \mathrm{mM} \mathrm{Mg}^{2+}$, no chelators added, $\mathrm{pH}$ 7.3) saline. This was necessary because marked and variable effects upon measured electrical coupling coefficients $\left(V_{2} / V_{1}\right.$; Bennett, 1966) were produced by conductance shunts resulting from chemical synaptic activity in standard physiological saline. As an example, the electrical coupling coefficient between neurons $4 \mathrm{R}$ and $4 \mathrm{~L}$ in one preparation increased from 0.3 in standard saline to 0.6 in low $\mathrm{Ca}^{2+} / \mathrm{high} \mathrm{Mg}^{2+}$ saline. The average response from three to four current pulses was taken as the coupling coefficient measurement.

Neuronal morphology and the presence of dye coupling were ascertained by intracellular iontophoresis of $3 \%$ (in distilled water) Lucifer Yellow CH (Stewart, 1978, 1981). Dye was injected by DC hyperpolarizing currents of 4 to $10 \mathrm{nA}$ for 2 to $6 \mathrm{~min}$. Because the relationship of current injection to dye injection was somewhat variable, the injected neuron was then observed by inserting a blue filter over the tungsten lamp of the stereomicroscope (Kater and Hadley, 1982). If the injected neuron's soma did not appear bright yellow, additional DC current (4 to $5 \mathrm{nA}$ ) was injected for up to $10 \mathrm{~min}$. If the soma did not appear bright yellow at this time the preparation was discarded. Specimens were incubated in saline for 20 to 90 min following dye injection, then fixed overnight in phosphate-buffered $4 \%$ formaldehyde $\left(\mathrm{pH} \mathrm{7.4)}\right.$ at $4^{\circ} \mathrm{C}$. They were then dehydrated through an alcohol series, cleared with methyl salicylate, mounted on glass slides, and viewed and photographed through a Zeiss Universal microscope with epi-illumination. This procedure consistently yielded bright stains of the injected neurons. Dye coupling was considered positive if there was fluorescence in the uninjected neuron 4 soma which was detectable above the nonspecific background fluorescence in the ganglion.

Statistical comparisons. Mean coupling coefficients $( \pm$ SD) for the different experimental groups are given in the text. The standard deviations illustrate the variability of coupling values within a group. In addition, $95 \%$ confidence limits (CLs) for comparisons of the means were calculated by taking $\tau_{50} \times$ SEM. Differences between experimental groups were considered significant if the mean of each group fell outside the $95 \%$ confidence limit of the other group (cf. Sokal and Rohlf, 1981).

\section{Results}

Morphology and connectivity of neurons $4 R$ and $4 L$. Neurons 4R and 4L (map of Kater and Rowell, 1973) in the buccal ganglia of Helisoma have been the focus of a number of previous investigations (Kater, 1974; Merickel and Kater, 1974; Kater et al., 1978; Murphy and Kater, 1978, 1980a, b; Bahls et al., 1980; Bulloch and Kater, 1982; Hadley and Kater, 1983). These electrically coupled neurons are a mirror image pair (Fig. 1), each sending an

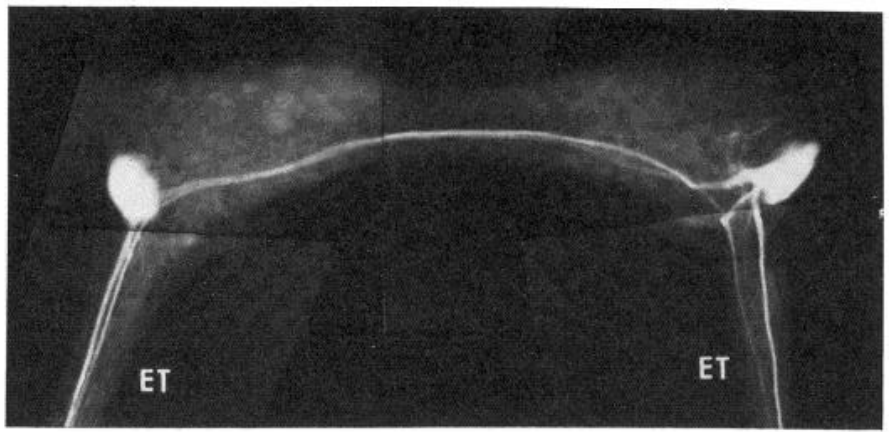

Figure 1. Morphology of neurons 4R and 4L. Both 4R and $4 \mathrm{~L}$ were injected with Lucifer Yellow $\mathrm{CH}$. Each sends an axon out the ipsilateral esophageal trunk $(E T)$ and a second axon across the buccal commissure and out the contralateral ET. Each neuron has a dendritic tree in the ipsilateral neuropile and usually a much smaller arborization in the contralateral neuropile. There is sometimes a short neuritic process extending directly from the soma of one neuron 4 to the nearby axon of the contralateral neuron 4. 
axon into the ipsilateral esophageal nerve trunk (ET) and a second axon across the buccal commissure and into the contralateral ET. Careful observation under high magnification of preparations with both neurons dyefilled revealed that axons of the two neurons came into close apposition only at locations within the neuropile near their respective somata. These locations are the presumed sites of electrical communication.

Simultaneous intracellular recordings from neurons $4 \mathrm{R}$ and $4 \mathrm{~L}$ showed that current injected into one of the neurons resulted in a voltage displacement in both neurons. The mean electrical coupling coefficient (postsynaptic/presynaptic voltage ratio) in low $\mathrm{Ca}^{2+}$, high $\mathrm{Mg}^{2+}$ saline was $0.54 \pm 0.11(n=13 ; 95 \% \mathrm{CL}=0.07)$.

Effects of axotomy upon coupling characteristics. Axotomy of neurons $4 \mathrm{R}$ and $4 \mathrm{~L}$ evoked a significant increase in the mean electrical coupling coefficient from 0.54 in normal ganglia to $0.72 \pm 0.14(n=24 ; 95 \% \mathrm{CL}=0.06)$ in buccal ganglia with axotomized neurons cultured 3 to 5 days in blood (Fig. 2). Such differences in coupling have significant physiological consequences. The efficacy of presynaptic action potentials for evoking postsynaptic action potentials is much greater in more closely coupled pairs of neurons (Fig. 3).

The axotomy-induced increase in coupling can be seen as early as $48 \mathrm{hr}$ after axotomy but is not present just after nerve crush. One to four hours following axotomy there was a slight but significant decrease in coupling coefficients $(\overline{\mathrm{X}}=0.45 \pm 0.09 ; n=8 ; 95 \% \mathrm{CL}=0.07)$. Measurements made on the same pairs of neurons approximately $1 \mathrm{hr}$ after axotomy and again after 3 days in culture confirmed that there was a near doubling in coupling coefficients during the interval between meas-

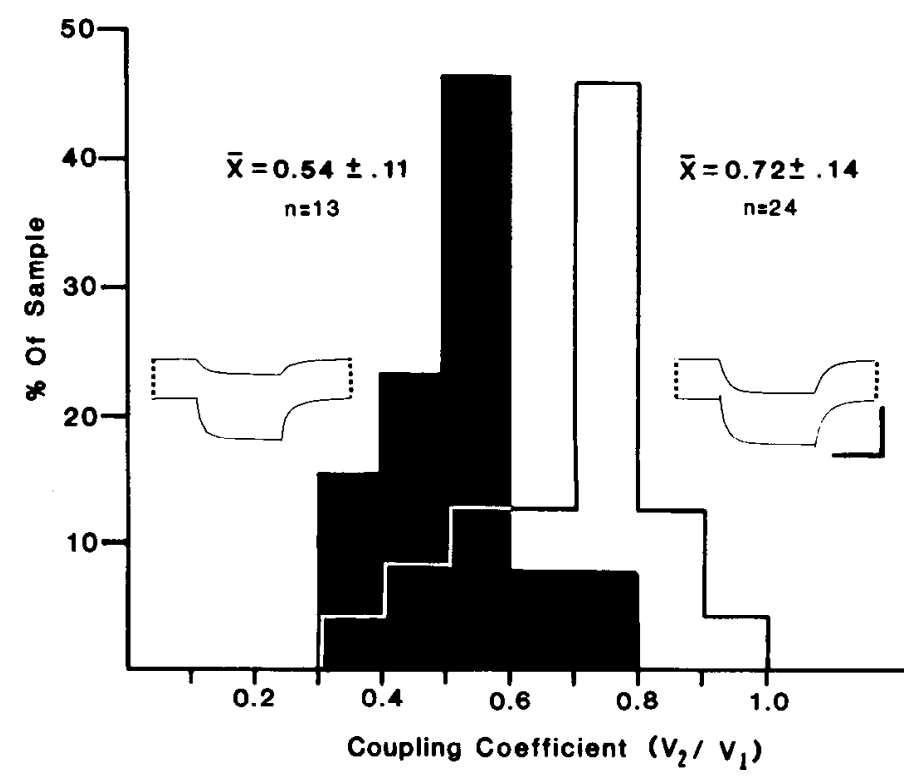

Figure 2. Distribution of coupling coefficients of neurons 4R and $4 \mathrm{~L}$ from normal preparations and following axotomy. The solid histogram plots coupling coefficient versus percentage of sample for normal pairs of neurons $4 \mathrm{R}$ and $4 \mathrm{~L}$. The open histogram shows the increase in coupling coefficients following axotomy of neurons $4 \mathrm{R}$ and $4 \mathrm{~L}$ and subsequent culture in blood for 3 to 5 days. Means for each sample are given \pm SD. Insets display representative coupling measurements. Calibrations: $20 \mathrm{mV}$; left inset, $1 \mathrm{sec}$; right inset, $400 \mathrm{msec}$.
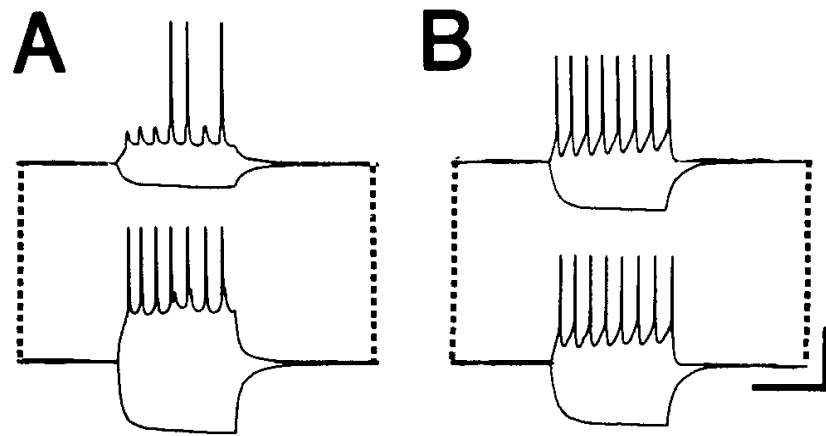

Figure 3. Physiological consequences of variable electrical coupling. $A$, Simultaneous recordings from a relatively weakly coupled pair of neurons $4\left(V_{2} / V=0.35\right)$ show frequent failure of transmission of action potentials in the "postsynaptic" neuron (upper trace) when depolarizing current is injected into the other neuron (lower trace). $B$, A relatively strongly coupled pair of neurons $4\left(V_{2} / V_{1}=0.70\right)$ displays $1 / 1$ following of action potentials when current is injected into one of the neurons. The peaks of action potentials in both $A$ and $B$ were clipped on the chart recordings. Calibrations: $20 \mathrm{mV}, 2$ sec.

urements. Hence the loss of possible axonal current sinks as a result of axotomy is not the cause of coupling changes, and the axotomy-induced increases in electrical coupling are time dependent.

Possible effects of the in vitro culture procedure per se upon electrical coupling were examined. After 3 to 5 days in culture, preparations with intact neuron 4 connections onto salivary glands, and preparation consisting of isolated buccal ganglia with long, uncrushed esophageal nerve trunks (i.e., axons of neurons $4 \mathrm{R}$ and $4 \mathrm{~L}$ were severed near the salivary glands at a distance of greater than $1 \mathrm{~mm}$ from the normal sites of electrical coupling) showed no difference in coupling coefficients $(\overline{\mathrm{X}}=0.54$ $\pm 0.06 ; n=7 ; 95 \% \mathrm{CL}=0.05$ ) from normal preparations. This ruled out direct effects of the culture conditions upon electrical coupling.

These observations, taken together, show that axotomy at a site near the buccal ganglia ultimately caused a delayed enhancement of the electrical coupling between neurons $4 \mathrm{R}$ and $4 \mathrm{~L}$.

Dye coupling and its correlation with electrical coupling between neurons $4 R$ and $4 L$. Dye coupling (cf. Stewart, 1978, 1981) between neurons $4 \mathrm{R}$ and $4 \mathrm{~L}$ was used as a further probe of communication between these neurons in both normal and axotomized preparations. Nonjunctional shunt resistances affect electrical coupling coefficient measurements and, in this system, non-isopotentiality precludes either voltage or current clamp measurements of junctional conductance. Therefore, an independent assessment of junctional conductance was desirable. Thus, dye coupling measurements were used to ascertain whether axotomy-induced changes in the electrical coupling coefficient reflected junctional changes in the permeation of molecules of a size expected to permeate gap junctions (Simpson et al., 1977).

Dye coupling (Fig. 4) was observed in only $27 \%(n=$ 27) of neuron 4 pairs in normal ganglia even though electrical coupling was observed in all preparations. Thus dye coupling in normal ganglia was not well correlated with the presence of electrical coupling. However, the increases in electrical coupling evoked by axotomy were 
associated with parallel increases in the incidence of dye coupling. Eighty-seven percent $(n=15)$ of the axotomized preparations cultured 3 to 5 days in blood were dye coupled. The intensity of fluorescence in the uninjected neuron 4 displayed parallel changes. With higher coupling coefficients, there was often noticeably more intense fluorescence in the uninjected neuron 4 than in less highly coupled pairs. Dye coupling in normal ganglia was always faint; that is, dye was visible only in the soma of the uninjected neuron with no visible axonal conduit between the axon of the injected neuron and the soma of the uninjected neuron (Fig. $5 A$ ). However, the intensity of fluorescence seen in the uninjected neuron 4 of axotomized preparations was sometimes much greater than that ever seen in normal ganglia, such that an uninjected member of a pair could have not only the soma but both the commissural and esophageal trunk axons visible (Fig. $5 B$ ). This clear difference in intensity of fluorescence in uninjected dye-coupled neurons served to reinforce quantitative observations on changes in electrical coupling following axotomy.

The most important evidence that increases in electrical and dye coupling reflected a common mechanism was the consistent relationship seen between the magnitude
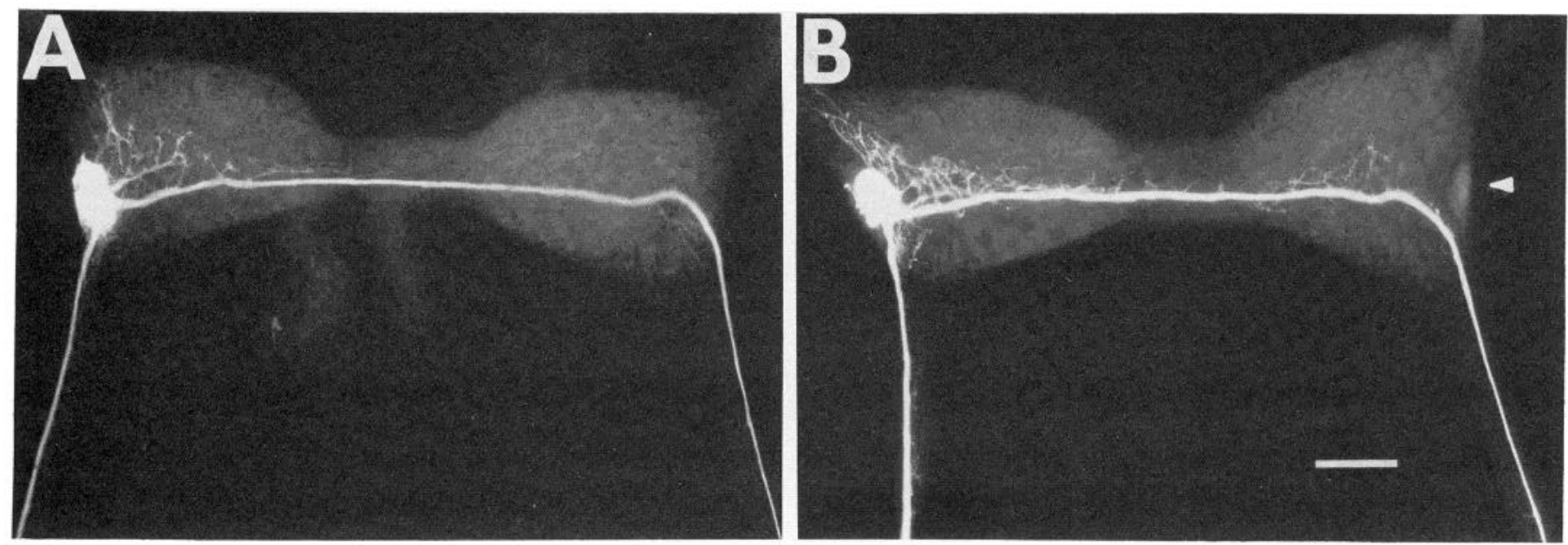

Figure 4. Dye coupling between neurons 4R and 4L. Lucifer Yellow $\mathrm{CH}$ was injected into a single neuron 4 of each preparation. $A$, In $73 \%$ of the preparations no dye was visible in the contralateral neuron. The coupling coefficient for the preparation shown was 0.47 . B, A similar preparation which did display dye coupling (fluorescence in the uninjected contralateral neuron 4 soma; arrowhead). The 4-4 coupling coefficient for this preparation was 0.58 . Calibration bar, $100 \mu \mathrm{m}$.
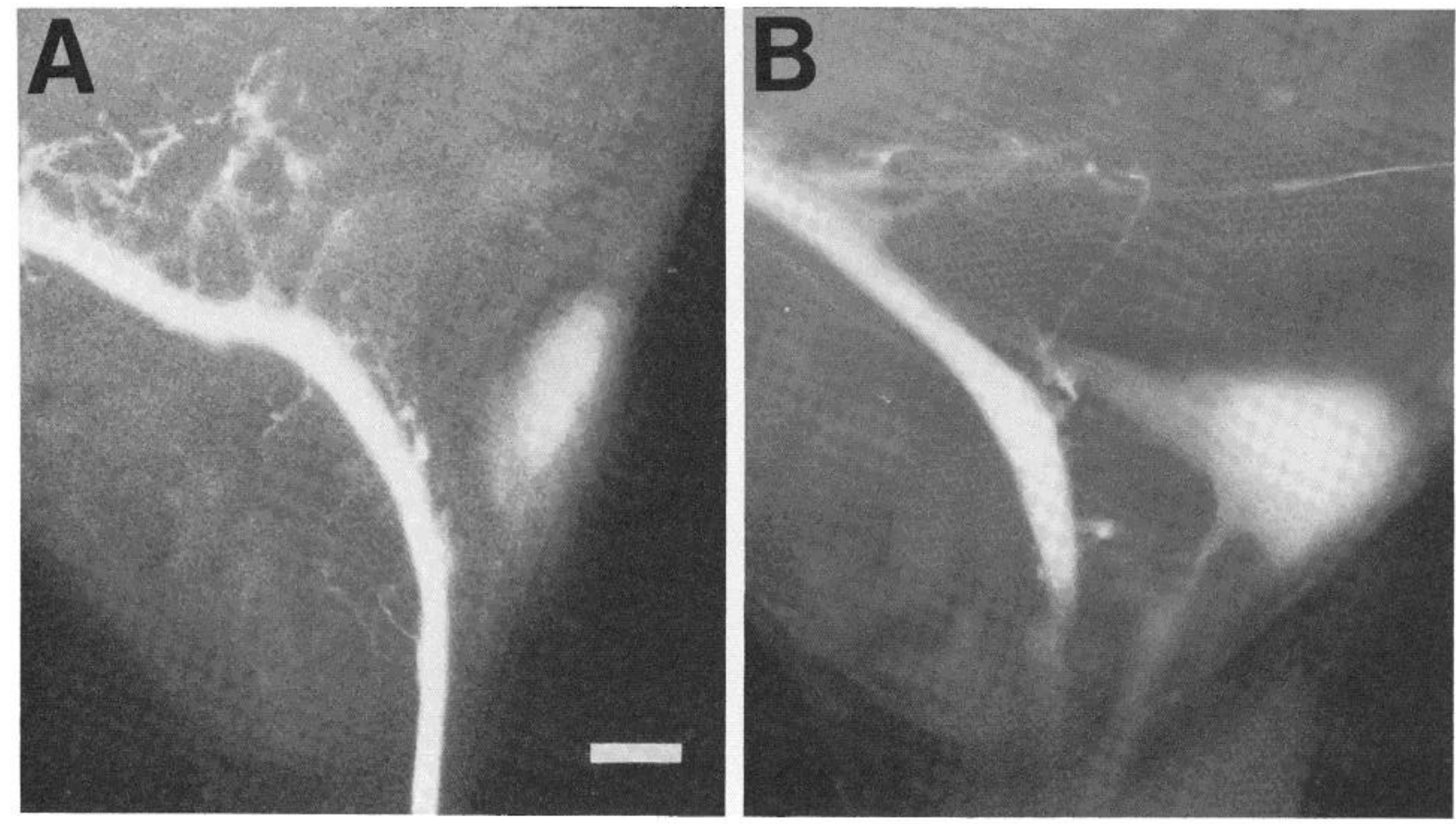

Figure 5. Differences in intensity of dye coupling. $A$, Higher magnification of the corner of the contralateral ganglion from Figure $4 B$ showing that only the soma of the uninjected neuron has visible fluorescence. $B$, Following axotomy and culture, not only the probability of dye coupling increases (see the text) but also the intensity of fluorescence in the uninjected neuron 4 is often greater than ever observed in normal preparations. Here both the soma and the ipsilateral and commissural axons of the uninjected neuron can be seen. Calibration bar, $25 \mu \mathrm{m}$. 
of electrical coupling and the probability of detectable dye coupling (Fig. 6). Dye coupling was never detected when electrical coupling coefficients between neuron pairs were less than $0.50(n=13)$. On the other hand, all preparations were dye coupled when coupling coefficients were greater than $0.70(n=9)$. Intermediate coupling coefficients (greater than 0.50 but less than 0.70 ) were associated with an intermediate probability of dye coupling $(67 \% ; n=15)$. Thus dye coupling is correlated not with the presence or absence but with the magnitude of electrical coupling, and there is an apparent "threshold of detectability" for dye coupling associated with a coupling coefficient value slightly higher than 0.50 . Accordingly, most neuron 4 pairs from normal ganglia have coupling coefficients lower than the threshold for detectable dye coupling, and most axotomized neuron 4 pairs have electrical coupling values above the threshold for detection of dye passage. As expected, the mean coupling coefficient of $4 \mathrm{R}$ and $4 \mathrm{~L}$ in those normal animals with detectable dye coupling was statistically greater $(\overline{\mathrm{X}}=$ $0.64 \pm 0.08 ; n=5 ; 95 \% \mathrm{CL}=0.10)$ than that in preparations with no detectable dye coupling $(\mathrm{X}=0.48 \pm 0.08$; $n=8 ; 95 \% \mathrm{CL}=0.07$ ). In addition, dye-coupled axotomized pairs of neuron 4 had an average coupling coefficient of $0.78 \pm 0.11(n=10 ; 95 \% \mathrm{CL}=0.08)$, while the two axotomized pairs that failed to display dye coupling had

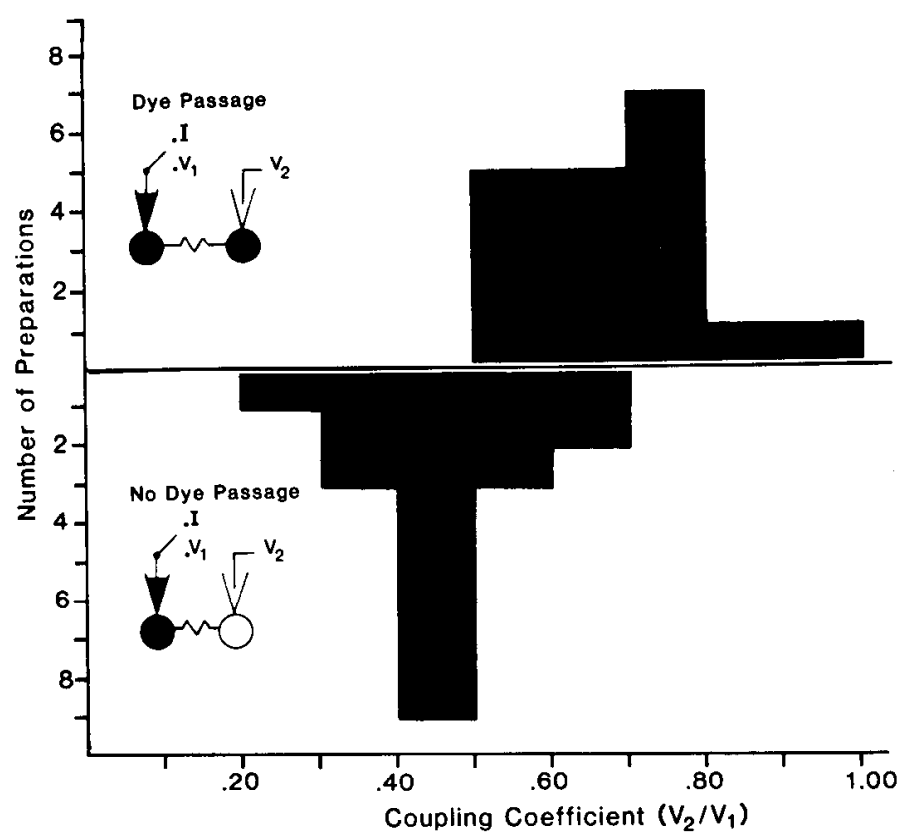

Figure 6. Relationship between dye coupling and electrical coupling coefficient. The upper histogram (above the horizontal line) gives the distribution of electrical coupling coefficients for all preparations that displayed dye coupling between neurons $4 \mathrm{R}$ and $4 \mathrm{~L}$ and for which electrical measurements were made $(n=19)$. The lower histogram displays the distribution of all preparations which did not show dye coupling $(n=18)$. No preparations with $4 \mathrm{R}-4 \mathrm{~L}$ coupling coefficients $<0.5$ displayed dye coupling, all with coupling coefficients $>0.7$ displayed dye coupling, and $67 \%$ of those with coupling coefficients between 0.5 and 0.7 displayed dye coupling. Normal preparations and those with all experimental manipulations (i.e., acute axotomy, axotomy and culture, and culture without axotomy) were included here. coupling coefficients of 0.55 and 0.68 . Taken together, these data on electrical and dyc coupling in the same neuron 4 pairs confirmed the intuitive expectation that greater electrical coupling would be associated with a greater probability of dye coupling.

\section{Discussion}

The present demonstration of axotomy-induced increases in electrical and dye coupling must be viewed in the context of the numerous other axotomy-evoked changes in identified neurons of Helisoma. We have described previously a series of phenomena including: (1) extensive neuritic outgrowth which produces predictable alterations in morphology (Murphy and Kater, 1978, 1980a, b; Bulloch and Kater, 1981, 1982; Hadley et al., 1982; Stamler et al., 1982); (2) reinnervation of appropriate peripheral targets by regenerating axons (Murphy and Kater, 1978, 1980a); (3) transient reduction in efficacy of some chemical inhibitory postsynaptic potentials (Bulloch et al., 1980); (4) de novo formation of stable or transient electrical synapses (Bulloch and Kater, 1981, 1982; Hadley et al., 1982). These changes are not independent events; rather some may be permissively or causally related to others. For instance, the formation of connections between identified neurons $5 \mathrm{R}$ and $5 \mathrm{~L}$ must be preceded by neuritic growth to allow contact between the spatially separate neurons (Bulloch and Kater, 1982). Some changes (e.g., neuritic outgrowth) are characteristic of all examined axotomized Helisoma neurons, while other plastic events (e.g., pathfinding; Murphy and Kater, $1980 \mathrm{~b})$ are specific to particular identified neurons. The set of responses of a given neuron together may be considered to be the "axotomy response" of that neuron (Cohan et al., 1983). A new facet of the axotomy response of electrically coupled Helisoma neurons appears to be that existing intercellular electrotonic communication is strengthened (Fig. 2; see also Bulloch et al., 1980, for indirect indications of a similar phenomenon between other neurons in Helisoma).

Possible mechanisms of coupling changes. The axotomy-induced increases in electrical and dye coupling could result from any event which effectively reduced junctional resistance or increased nonjunctional resistance to both ions and dye (cf. Bennett, 1966). Axotomy per se could be the proximate cause of an increase in coupling if the axons of neuron 4 represented a significant "sink" for ions or dye molecules. Severance of these axons might (after sealing) result in an increase in nonjunctional resistance to both ions and dye. However, axotomy had little short-term effect upon coupling coefficients. Thus, the immediate effects of axotomy in removing axonal "sinks" for ionic current could not be the proximate cause of the observed increase in coupling following axotomy and culture. Additionally, since culture itself had no effect upon coupling, time- and axotomy-dependent alterations must be necessary for this dynamic modulation of synaptic efficacy.

Although the ultrastructural bases of both normal and enhanced electrical coupling have not been investigated, previous physiological evidence suggests that coupling between neurons $4 \mathrm{R}$ and $4 \mathrm{~L}$ may be mediated by gap junctions. Reduced cytoplasmic $\mathrm{pH}$ has been shown to 
reversibly reduce electrical coupling mediated by gap junctions in several systems (Turin and Warner, 1977; Iwasuki and Peterson, 1979; Spray et al., 1981). Bulloch and Kater (1982) recently showed that lowering $\mathrm{pH}$ can also reversibly reduce normally extant electrical coupling between neurons $4 \mathrm{R}$ and $4 \mathrm{~L}$ in Helisoma as well as the newly formed connections between axotomized neurons $5 \mathrm{R}$ and $5 \mathrm{~L}$. It therefore seems plausible that the increased communication reported here is the result of an increase in gap junctional area (i.e., an increase in the number of junctional channels). An increase in gap junctional area could represent an expansion of existing junctions or the formation of new junctions at new sites (e.g., by newly formed neurites; Bulloch and Kater, 1982; see also Taghert et al., 1982). The alternative possibility that increased electrical communication might reflect an increase in the unit conductance of extant junctional channels has not been ruled out.

Correlation of electrical and dye coupling. The relationship between electrical and dye coupling often has seemed capricious. Dye coupling frequently is not seen (or is seen only in a fraction of preparations) between cells known to be electrically coupled (e.g., Bennett et al., 1972, 1978; Kaneko et al., 1978; Lo and Gilula, 1979; Audesirk et al., 1982; Warner and Lawrence, 1982). Assuming the involvement of gap junctions (cf. Bennett and Goodenough, 1978; Loewenstein, 1979) there are two reasonable explanations for the failure of dye coupling in electrically coupled systems. (1) Individual junctional channels or pores may allow ionic current to flow but may prevent the passage of the larger dye molecules. (2) Although individual pores may allow conductance of dye molecules, the concentration of dye in the uninjected cell may fail to reach a detectable threshold (about $10^{-6} \mathrm{M}$ for Lucifer Yellow CH (Stewart, 1978); but the threshold will depend upon cell size and background fluorescence). The first explanation would imply an effective difference in pore size for those preparations that are dye coupled and those that are not. Although this is an interesting hypothesis (e.g., Warner and Lawrence, 1982), the question of the universality of junctional pore size remains problematical, since channel size has been probed systematically only in Chironomous salivary glands and vertebrate tissue culture cells (cf. Pitts and Simms, 1977; Loewenstein, 1979). On the other hand, evidence tends to support the second explanation. If individual junctional channels have a unit conductance for ions and dye molecules, then there should be a requisite correlation between electrical and dye coupling. Gap junctional area has been shown to covary with electrical and/or dye coupling in several systems (e.g., Novikoff hepatoma cells, Sheridan et al., 1978; normal and regenerating rat liver, Meyer et al., 1981; Patella embryos, Dorresteijn et al., 1982; and murine salivary glands, S. B. Kater and N. Galvin, unpublished observation). In those cases where electrical coupling but not dye coupling is observed, transferred dye could simply be below the threshold concentration for detection with the incubation times employed (cf. Bennett et al., 1978; Stewart, 1978). Similarly, the frequent detection of dye only in the soma of the "receiving" neuron and not in the axonal conduit (Figs. 4 and 5) suggests that the concentration of dye in the axon does not reach a detection threshold. However, a variety of factors could contribute to the preferential visualization of dye in the somata (e.g., the greater diameter of the somata relative to axons, and binding of dye to the nuclei; cf. Kater and Nicholson, 1973).

In an ideal system, electrical coupling coefficients and dye coupling should be correlated. However, electrical coupling coefficient values are greatly influenced by variable nonjunctional shunt conductances (e.g., from afferent synaptic activity). In addition, the sensitivity of methods for detecting dye movement at low concentrations may be less than that of methods for detection of electrical current. Thus the correlation between electrical and dye coupling in a particular preparation could easily go undetected, especially if the range of coupling coefficients is small and the degree of coupling in most preparations is below the threshold for detection of dye coupling. The apparent capriciousness of the relationship between electrical and dye coupling in Helisoma neurons $4 \mathrm{R}$ and $4 \mathrm{~L}$ disappeared as the range of coupling coefficients was expanded by obtaining the larger values from axotomized preparations. Sufficient numbers of measurements of electrical and dye coupling (with chemical synaptic activity blocked) revealed the expected result that detectable dye premeation was associated with the higher electrical coupling coefficients.

Malleability of electrical communication. Clearly, electrotonic connections are not rigid relays. Physiological modulation of shunt conductances can regulate the efficacy of such connections (cf. Spira et al., 1976; Spray et al., 1979; Zipser, 1979). In addition, Rayport and Kandel (1980) have shown a functional uncoupling between identified neurons of Aplysia during development which results from growth of the axons and increased shunting of synaptic current. The shunting overrides a concurrent increase in synaptic conductance. There are numerous other cases of plasticity of electrical communication during development (see Bennett et al., 1981, for review). In addition, adult cells undergo modification in electrical communication associated with hormonal changes (e.g., Garfield et al., 1978; Browne et al., 1979; Garfield et al., 1980), and perhaps also during transformation of cancerous cells (e.g., Loewenstein, 1979). This report shows a rapid increase in neuronal electrical communication following axotomy. Perhaps such plastic events in electrically coupled systems reflect similar processes occurring on a smaller scale during normal steady-state activity.

\section{References}

Audesirk, G., T. Audesirk, and P. Bowsher (1982) Variability and frequent failure of Lucifer Yellow to pass between two electrically coupled neurons in Lymnaea stagnalis. J. Neurobiol. 13: 369-375.

Bahls, F., S. B. Kater, and R. W. Joyner (1980) Neuronal mechanism for bilateral coordination of salivary gland activity in Helisoma. J. Neurobiol. 11: 365-379.

Bennett, M. V. L. (1966) Physiology of electrotonic junctions. Ann. N. Y. Acad. Sci. 137: 509-539.

Bennett, M. V. L., and D. A. Goodenough (1978) Gap junctions, electrotonic coupling, and intercellular communication. Neurosci. Res. Program Bull. 16: 373-486.

Bennett, M. V. L., M. E. Spira, and G. D. Pappas (1972) 
Properties of electrotonic junctions between embryonic cells of Fundulus. Dev. Biol. 29: 419-435.

Bennett, M. V. L., M. E. Spira, and D. C. Spray (1978) Permeability of gap junctions between embryonic cells of Fundulus: A reevaluation. Dev. Biol. 65: 114-125.

Bennett, M. V. L., D. C. Spray, and A. L. Harris (1981) Electrical coupling in development. Am. Zool. 21: 413-427.

Browne, C. L., H. S. Wiley, and J. N. Dumont (1979) Oocytefollicle cell gap junctions in Xenopus Laevis and the effects of gonadotropin on their permeability. Science 203: 182-183.

Bulloch, A. G. M., and S. B. Kater (1981) Selection of a novel connection by adult molluscan neurons. Science 212: 79-81.

Bulloch, A. G. M., and S. B. Kater (1982) Neurite outgrowth and selection of new electrical connections by adult Helisoma neurons. J Neurophysiol. 48: 569-583.

Bulloch, A. G. M., S. B. Kater, and A. D. Murphy (1980) Connectivity changes in an isolated molluscan ganglion during in vivo culture. J. Neurobiol. 11: 531-546.

Cohan, C. S., R. D. Hadley, and S. B. Kater (1983) "Zap axotomy:" Localized fluorescent excitation of single dye-filled neurons induces growth by selective axotomy. Brain Res., in press.

DeVoogd, T., and F. Nottebohm (1981) Gonadal hormones induce dendritic growth in the adult avian brain. Science 214: 202-205.

Dorresteijn, A. W. C., S. M. Biliński, J. A. M. van den Biggelaar, and J. G. Bluemink (1982) The presence of gap junctions during early Patella embryogenesis: An electron microscopical study. Dev. Biol. 91: 397-401.

Garfield, R. E., D. Merrett, and A. K. Grover (1980) Gap junction formation and regulation in myometrium. Am. J. Physiol. 239: C217-C228.

Garfield, R. E., S. M. Sims, M. S. Kannan, and E. E. Daniel (1979) Possible role of gap junctions in activation of myometrium during parturition. Am. J. Physiol. 235: C168-C179.

Granzow, B. (1979) Identified higher-order neurons which regulate the feeding motor program of Helisoma trivolvis. Ph.D. Thesis, University of Iowa, Iowa City, IA.

Hadley, R. D., and S. B. Kater (1983) Competence to form electrical connections is restricted to growing neurites in the snail, Helisoma. J. Neurosci. 3: 924-932.

Hadley, R. D., R. G. Wong, S. B. Kater, D. L. Barker, and A. G. M. Bulloch (1982) Formation of novel central and peripheral connections between molluscan central neurons in organ cultured ganglia. J. Neurobiol. 13: 217-230.

Iwasuki, N., and O. H. Peterson (1979) Pancreatic acinar cells: the effect of carbon dioxide, ammonium chloride and acetylcholine on intercellular communication. J. Physiol. (Lond.) 291: 317-326.

Kandel, E. R. (1976) Cellular Basis of Behavior, An Introduction to Behavioral Neurology. Freeman, San Francisco.

Kaneko, C. R. S., M. Merickel, and S. B. Kater (1978) Centrally programmed feeding in Ilelisoma: Identification and characteristics of an electrically coupled premotor neuron network. Brain Res. 146: 1-21.

Kater, S. B. (1974) Feeding in Helisoma trivolvis, the morphological and physiological basis of a fixed action pattern. Am. Zool. 14: 1017-1036.

Kater, S. B. and R. D. Hadley (1982) Intracellular staining combined with video fluorescence microscopy for viewing living identified neurons. In Cytochemical Methods in Neuroanatomy. S. L. Palay and V. Chan-Palay, eds., pp. 441-459, A. R. Liss, Inc., New York.

Kater, S. B., and C. R. S. Kaneko (1972) An endogenously bursting neuron in the gastropod mollusc, Helisoma trivolvis: characterization of activity in vivo. J. Comp. Physiol. 79: 114.

Kater, S. B. and C. Nicholson (1973) Intracellular Staining in Neurobiology. Springer-Verlag, New York.
Kater, S. B., and C. H. F. Rowell (1973) Integration of sensory and centrally programmed components in generation of cyclical feeding activity in Helisoma trivolvis. J. Neurophysiol. 36: 142-155.

Kater, S. B., A. D. Murphy, and J. Rued (1978) Control of the salivary glands of Helisoma by identified neurons. J. Exp. Biol. 72: 91-106.

Levine, R. B., and J. W. Truman (1982) Metamorphosis of the insect nervous system: changes in morphology and synaptic interactions of identified neurones. Nature 299: 250-252.

Lo, C. W., and N. B. Gilula (1979) Gap junctional communication in the postimplantation mouse embryo. Cell 18: 411-422.

Loewenstein, W. R. (1979) Junctional intercellular communication and the control of growth. Biochim. Biophys. Acta 560: 1-65.

Merickel, M. B., E. D. Eyman, and S. B. Kater (1977) Analysis of a network of electrically coupled neurons producing rhythmic activity in the snail Helisoma trivolvis. IEEE Trans. Biomed. Eng. 24: 277-287.

Merickel, M., and S. B. Kater (1974) Neuronal change: Compensatory acclimation of the contribution of an electrogenic pump to the resting potential. J. Comp. Physiol. 94: 195-206.

Meyer, D. J., S. B. Yancey, and J. -P. Revel (1981) Intercellular communication in normal and regenerating rat liver: A quantitative analysis. J. Cell Biol. 91: 505-519.

Murphy, A. D., and S. B. Kater (1978) Specific reinnervation of a target organ by a pair of identified molluscan neurons. Brain Res. 156: 322-328.

Murphy, A. D., and S. B. Kater (1980a) Sprouting and functional regeneration of an identified neuron in Helisoma. Brain Res. 186: 251-272.

Murphy, A. D., and S. B. Kater (1980b) Differential discrimination of appropriate pathways by regenerating identified neurons in Helisoma. J. Comp. Neurol. 190: 395-403.

Nottebohm, F. (1981) A brain for all seasons: Cyclical anatomical changes in song control nuclei of the canary brain. Science 214: 1368-1370.

Pitts, J. D., and J. W. Simms (1977) Permeability of junctions between animal cells. Intercellular transfer of nucleotides but not of macromolecules. Exp. Cell Res. 104: 153-163.

Rayport, S. G., and E. R. Kandel (1980) Developmental modulation of an identified electrical synapse: Functional uncoupling. J. Neurophysiol. 44: 555-567.

Sheridan, J. D., M. Hammer-Wilson, D. Preus, and R. G. Johnson (1978) Quantitative analysis of low-resistance junctions between cultured cells and correlation with gap-junctional areas. J. Cell Biol. 76: 532-544.

Simpson, I., B. Rose, and W. R. Loewenstein (1977) Size limit of molecules permeating the junctional membrane channels. Science 195: 294-296.

Sokal, R. R. and F. J. Rohlf (1981) Biometry. Freeman, San Francisco, 859 pp.

Spira, M. E., D. C. Spray, and M. V. L. Bennett (1976) Electrotonic coupling: effective sign reversal by inhibitory neurons. Science 194: 1065-1067.

Spray, D. C., A. L. Harris, and M. V. L. Bennett (1979) Voltage dependence of junctional conductance in early amphibian embryos. Science 204: 432-434.

Spray, D. C., A. L. Harris, and M. V. L. Bennett (1981) Gap junctional conductance is a simple and sensitive function of intracellular $\mathrm{pH}$. Science 211: 712-715.

Stamler, J. F., S. B. Kater, A. D. Murphy, and A. G. M. Bulloch (1982) Neuritic growth and neuroma formation by an isolated molluscan ganglion. J. Neurobiol. 13: 85-89.

Stewart, W. W. (1978) Functional connections between cells as revealed by dye-coupling with a highly fluorescent naphthalimide tracer. Cell 14: 741-759.

Stewart, W. W. (1981) Lucifer dyes-Highly fluorescent dyes for biological tracing. Nature 292: 17-21. 
Taghert, P. H., M. J. Bastiani, R. K. Ho, and C. S. Goodman (1982) Guidance of pioneer growth cones: Filopodial contacts and coupling revealed with an antibody to Lucifer Yellow. Dev. Biol. 94: 391-399.

Turin, L., and A. Warner (1977) Carbon dioxide reversibly abolishes ionic communication between cells of early am- phibian embryo. Nature 270: 56-57.

Warner, A. E., and P. A. Lawrence (1982) Permeability of gap junctions at the segmental border in insect epidermis. Cell 28: $243-252$.

Zipser, B. (1979) Voltage-modulated membrane resistance in coupled leech neurons. J. Neurophysiol. 42: 465-475. 